\title{
OPTIMIZATION OF AVERAGE DISTANCE BASED SELF-RELOCATION ALGORITHM USING AUGMENTED LAGRANGIAN METHOD
}

\author{
Shivani Dadwal ${ }^{1}$ and T. S. Panag ${ }^{2}$ \\ ${ }^{1}$ Deptt of Electronics and Communication Engineering, BBSBEC, \\ Fatehgarh Sahib, India \\ shivanidadwal @gmail.com \\ ${ }^{2}$ Deptt of Electronics and Communication Engineering, BBSBEC, \\ Fatehgarh Sahib, India \\ tripatjot.singh@bbsbec.ac.in
}

\begin{abstract}
Mobile robots with sensors installed on them are used in wireless sensor networks to generate information about the area. These mobile robotic sensors have to relocate themselves after initial location in the field to gain maximum coverage The average distance based algorithm for relocation process of mobile sensors does not require any GPS system for tracking the robotic sensors, thus avoiding cost, but increasing energy consumption. Augmented Lagrangian method is introduced in average distance based algorithm to reduce the extra energy consumption by sensors in average distance based relocation process. This modified average distance relocation scheme also improves the coverage area and the time taken by mobile robotic sensors to come to their final positions.
\end{abstract}

\section{KEYWORDS}

Average distance based self-relocation, modified average distance self-relocation.

\section{INTRODUCTION}

A lot of work has been done on sensor network as it has a wide application in various fields. One of its major application is in military area wherein surveillance of enemy areas has to be done. Earlier static sensors were deployed for detecting targets in a particular area. This reduced the coverage area of sensors. With advances in mobile robotics, now sensors can be carried by robotic structures. All they need is a direction which is administered by an algorithm. Execution of these algorithm leads to deployment of sensors in an area in a manner that maximum area is covered and all the targets are well detected. Even civilian application of such mobile sensors exists in property and homeland security $[1,2]$.

David C. Wyld et al. (Eds) : ACITY, DPPR, VLSI, WiMNET, AIAA, CNDC - 2015

pp. 193-203, 2015. @ CS \& IT-CSCP 2015

DOI : $10.5121 /$ csit.2015.51317 
For military purposes, the sensors are mostly dropped from air, which leads to a random deployment of sensors. These sensors have to reposition themselves. This issue has been covered in our paper. Optimization of coverage has been an active topic in sensor networks as along with coverage there are many other issues like minimum energy consumption and maximum lifetime which have to be kept in mind. Optimization of static sensor networks has been done using Genetic Algorithms which helps in locating sensors to their best position for maximum coverage and saves energy resulting in increased lifetime $[3,4,5,6]$. Other than this a few distributed and centralized algorithms have been introduced in an effort to modify and improve sensor networking [7]. The Genetic algorithm for optimization cannot be used for distributed algorithms as they require a central operating system to control the sensor positioning. Moreover Genetic algorithm can be used only where the area is well known.

Many algorithms have been developed for placing the sensors evenly in a field, out of which potential field algorithm is one [8]. In this algorithm, a potential field is generated by the sensors and they move accordingly. Another algorithm is the virtual force algorithm [9] in which the sensors move as per the attractive and repulsive forces generated by the sensors depending on the distances between them and attain their final positions. Some other methods namely, density control method [10] and fluid model based method [11] have also been introduced. There is another algorithm that repairs the coverage by finding the terminated sensors and moves these sensors to uncovered areas [12]. These algorithms listed above are applicable for distributed network where there is no central node and each sensor decides its own path. For this kind of network, a GPS system is must in order to track down the position of sensors after and before relocation. GPS is global positioning system that consumes a lot of power and is costly. Every time the sensor move during the execution of their respective algorithms, they send information to the GPS system which again consumes power.

Some methods were introduced to decrease the power consumption of sensor out of which one was to reduce the sensing range of sensor [13]. This was done on non-mobile sensors. It saves energy and increases the lifetime of sensors. The average distance based relocation process does not use any GPS system and hence cuts the energy consumption and cost as well [14]. But since there is no GPS used, the sensors consume extra energy to find their best final position which also increases the number of iterations to find the final coverage. Hence, optimization of average distance based self-relocation process is introduced to facilitate the sensors to find their final positions in less time which would also reduce energy consumption. This is done using augmented lagrangian optimization method [19]. In the next section, average distance based selfrelocation algorithm has been discussed.

\section{Average Distance BaSed Self Relocation Proceess}

\subsection{Assumptions}

Following are a few assumptions that we consider for the algorithm:

- Each sensor has a sensing area in the form of a circle, having radius, $r$. The probability of covering this area is 1 .

- The area A, where the sensors are randomly deployed is known by the sensors approximately 
- If the sensors come within their sensing range Rc, the strength of the signals transmitted by each sensor can be measured by the other.

- All sensors have certain range of communication and have a transmission power.

- Sensors have the ability to move as per the coordinates given after execution of algorithm.

- Sensors can detect obstacles in the field.

- Sensors that meet an obstacle in the field has its movement blocked and cannot communicate with the rest of sensors.

\subsection{Framework}

The main aim of this algorithm is designing a distributed algorithm which has a self- relocation capability to optimize the coverage area of field using less energy. It this algorithm, the distances between the sensors have to be known in order to relocate the sensors. The sensors transmit signal in the field once they are randomly deployed. These signals are intercepted by the sensors that come within the reach of .sensor areas of other sensors. The received signal strength is measured and corresponding distance is known.

Firstly, a "hello" signal is transmitted by the sensors which gives the signal strength to all the sensors, near or far, lying in sensor range. The distance corresponding to this signal strength is calculated by the sensors. Taking these distance information into account, the sensors move towards or away to each other and relocate themselves. Also, any obstacle coming in way has to be avoided by the sensors.

\section{METHODOLOGY}

\subsection{Ideal Deployment}

The ideal deployment is achieved when there are no spaces between the sensors. Such a condition is possible when the distance between the sensors is $\sqrt{3} r$ [15]. This is shown in Figure 1. Since it is not possible to achieve an ideal condition, in this algorithm, we try to achieve a near to ideal condition by placing sensors close. But as the field may have a deformity of obstacles, and various other factors, such a condition is difficult to achieve.

\subsection{Calculation of Threshold}

The total number of sensors in the field and the sensing field area are used to calculate the threshold distance dth and sensing radius that are near to ideal deployment. The threshold distance dth decides the sensor movement.

Let the total area of the sensor field be A. As shown in Figure 1, assume that each sensor has an effective area of coverage, $\mathrm{E}$ as in [14]. Let the total number of sensors deployed in the field be N. Effective coverage of each sensor is given as:

$$
E=\frac{A}{N}
$$


Also, E is a hexagonal area. Area of a hexagon is given as:

$$
E=\frac{3 \sqrt{3}}{2} \cdot r^{2}
$$

The threshold distance and sensing radius are calculated by the following equations:

$$
\begin{gathered}
r=\sqrt{\frac{2}{3 \sqrt{3}} \cdot \frac{A}{N}} \\
d_{t h}=\sqrt{3} r
\end{gathered}
$$

The effective coverage, E should be larger than this value, as the sensors which lie close to obstacles or to the edges will have less coverage. Hence, the threshold distance and sensor radius are increased by $15 \%$.

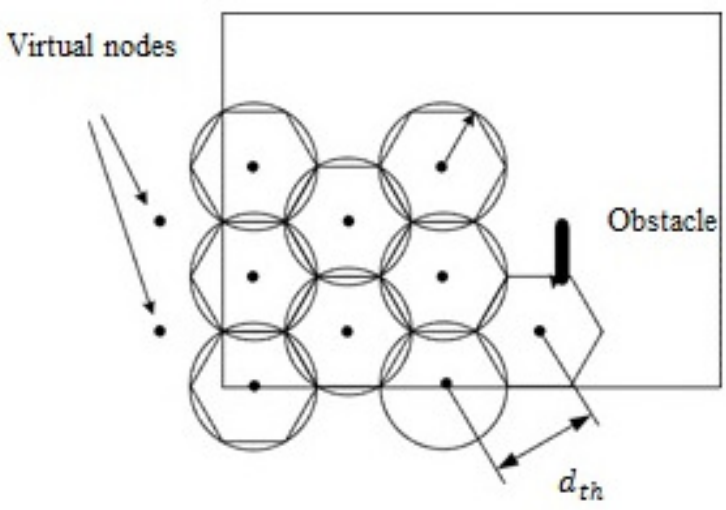

Figure 1. : Ideal coverage

\subsection{Virtual Nodes}

The algorithm considers that there exist virtual nodes at the boundary of the field. This is done to make deployment easy for the sensors lying close to the edges. Such virtual nodes do not actually exist. They are just used to avoid the sensors from getting closer to the edges. As shown in Figure 1, virtual nodes are considered along the boundary. No virtual nodes are required in the optimization technique used in this work.

\subsection{Movement Standards}

The sensors relocate themselves by adjusting the distances between themselves. They either move far or get closer to each other. No sensor has information about the direction of the other sensor. The criteria in which the sensors move is described as below:

Standard 1: If there is at least one sensor in the communication range of sensor $\mathrm{S}$ having distance less than $0.9 d_{t h}$, then the sensor will move away from other sensors.

Standard 2: If the standard 1 is not met and not more than 2 sensors lie at distance less than $1.1 d_{t h}$ from $\mathrm{S}$, then the sensor needs to move closer to other sensors.

Standard 3: Sensor S need not move if the above two standards are not met. 
A $10 \%$ margin is kept along these standards, so that the sensor is able to achieve a distance nearer to $d_{t h}$ from the rest of sensors.

\subsection{Moving Distance}

The movement of sensors is based on two standards, hence the moving distance is calculated by the following equation:

$$
d_{\text {travel }}= \begin{cases}d_{t h}-\frac{1}{m_{1}} \sum_{j=1}^{m_{1}} d_{j} & \text { for standard } 1 \\ \frac{1}{m_{2}} \sum_{i=1}^{m_{2}} d_{i}-d_{t h} & \text { for standard } 2 \\ 0 & \text { for standard } 3\end{cases}
$$

In the above equation, $d_{j}$ and $d_{i}$ are the distances from sensor $\mathrm{S}$ to other sensors. In the first standard, the sensor moves only for the sensors closer than distance threshold. The total number of sensors is given by $m_{l}$. In the second standard, all the neighboring sensors to sensor $\mathrm{S}$ are taken into account. The total number of sensors is $m_{2}$.

The direction of movement of the sensor is chosen randomly as the sensor is unaware of the neighbouring sensors. There can be a back and forth movement of sensors. To avoid this direction control scheme is used. As the sensors move, the difference of direction of movement is kept less than 90 degrees. Let the last direction of movement be $\alpha$, then in the next movement direction has to be in between $\alpha-90$ degrees to $\alpha+90$ degrees. When standard 1 is executed, the sensors move away from each other and when standard 2 is executed, the sensors move nearer to each other. As the sensors are moving to the direction chosen randomly, they check after moving through a short distance if the required coverage is attained. If not, they come back to their original positions.

\section{Simulation AND ANALYSIS}

For examining our results, we consider that the sensing field is a 100 by 100 grid structure. Each grid is 1 meter apart from the other grid. Consider that the sensing range of the sensors used lies within 18 to 25 meters. Hence, the maximum sensing radius is 25 meters. The range of communication of sensors is kept almost double the maximum sensing radius i.e. 55 meters.

\subsection{A Average Distance Based Self- relocation Algorithm Performance}

Let 20 sensors be randomly deployed in the sensor field. The distance threshold and sensing radius can be calculated from equations (3) and (4). A $15 \%$ increase should also be considered as explained in Section 2.3.

The following equations give the sensing radius and threshold distance of 20 sensors:

$$
\begin{aligned}
r & =1.15 \sqrt{\frac{2}{3 \sqrt{3}} \cdot \frac{A}{N}}=\sqrt{\frac{2}{3 \sqrt{3}} \frac{100 \times 100}{20}}=15.9 \text { meters } \\
d_{t h} & =\sqrt{3} r=\sqrt{3} \times 15.9=27.6 \text { meters }
\end{aligned}
$$


To analyze the results, three different conditions of initial sensor placement can be considered. In the first case, the sensors are all placed in the center of the sensing field such that they cover 50 by 50 meters area. In the second condition, the sensors are deployed or scattered in the whole sensing area. In the third condition, sensor are divided into 2 groups which are separately placed in the sensing field. The coverage initially is calculated for all the conditions which come out to be $55 \%, 50 \%$ and $61 \%$ approximately. The coverage can be calculated by the following equations:

$$
R_{\text {coverage }}=\frac{A_{\text {covered }}}{A_{\text {total }}}
$$

Here,

$R_{\text {coverage }}=$ coverage ratio

$A_{\text {covered }}=$ area covered by sensors cooperatively

$A_{\text {total }}=$ sensing field area

If we have to find out the coverage from the 100 by 100 grid structure, then the following equation can be used:

$$
R_{\text {coverage }}=\frac{n}{N}
$$

Here,

$\mathrm{n}=$ number of grid points covered by sensors

$\mathrm{N}=$ total number of grid points.

\subsection{Coverage Analysis}

The sensors in this algorithm do not have a fixed direction of movement. They move randomly and check if the required conditions are met. So, each of the three conditions stated in the above section are run 10000 times with a desired round number of 20. The execution results are shown in the Figure 2.

In figure 2, the results of average distance based algorithm are compared with virtual force algorithm. The virtual force algorithm is executed by taking its parameters into account as given in [16]. In all the three conditions, the coverage increases as the number of rounds increase. An average distance based self-relocation algorithm can achieve nearly $94 \%$ of coverage in the sensing field after 20 rounds. For a virtual force algorithm, after 20 rounds $93 \%$ coverage is achieved.

Thus, both the algorithm lead to almost same coverage of sensing field by the sensors. The major difference is that the average distance based algorithm does not require a GPS hardware, reducing the cost of the sensor network. It can hence be used in those areas where GPS cannot operate, like in under water systems. 


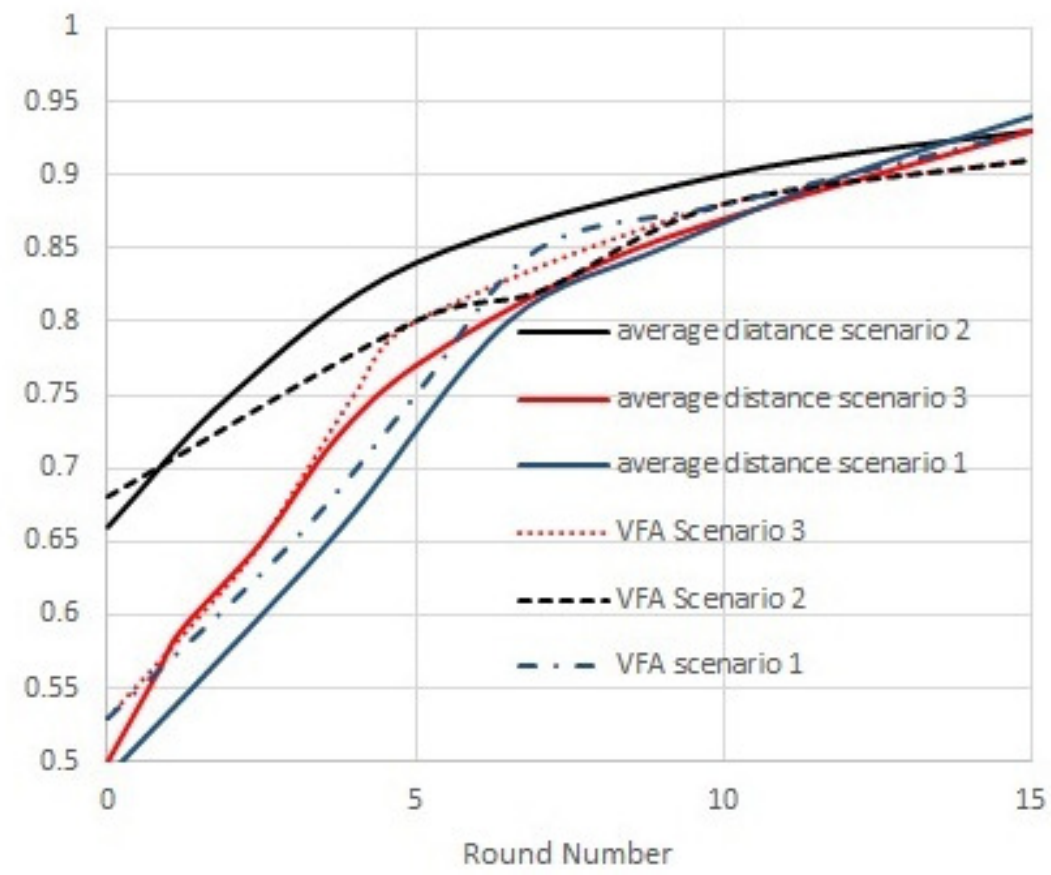

Figure 2: Simulation results of average coverage vs round number for average distance based self relocation algorithm

\subsection{Energy Analysis}

An energy analysis model has been discussed in [17]. This model has been used for mobile robots as per which energy consumed by a robot to move 1 meter is equal to 9.34 Joules if it is moving at a speed of $0.08 \mathrm{~m} / \mathrm{s}$ constantly. The amount of energy used by robots to turn by 90 degrees is 2.35 Joules. Both travelling and turning of mobile robots is considered to be at a constant speed. By doing so, we can plot a linear graph between energy consumed by mobile robot and coverage. As per the above discussion, we can divide energy consumption into two following parts:

- Energy used while travelling: If the mobile robot keeps moving at a constant rate in one direction, then the energy consumed for one single sensor node in a single round is given as:

$$
E_{\text {travel }}=d_{\text {travel }} \times 9.34 \text { (Joules) }
$$

- Energy used in direction changing: As discussed in the relocation algorithm, the sensor move randomly in some direction and checks its position by recalculating the signal strength. After this, it decides whether to move back to original position or not. Hence, during this process, the energy used by the sensor to turn in some direction is given as:

$$
E_{\text {turn }}= \begin{cases}\left(A_{\text {diff }} / 90\right) \times 2.35(\text { Joules }) & \text { Keep moivng } \\ (360 / 90) \times 2.35(\text { Joules }) & \text { Turning back }\end{cases}
$$

In the above equation, $A_{\text {diff }}$ is the difference of direction between the previous and later direction of sensor movement. If the new position does not satisfy the coverage requirement of sensor, then it gets back to position where it started by moving at 360 degrees, as shown in the above equation. 
To plot a graph, virtual force algorithm has also been considered. Its energy consumption is calculated. In the virtual force algorithm, the above two energy consumption are added to the energy used by the GPS system in locating the sensor nodes and in exchange of information between sensor and GPS. The GPS chip uses $198 \mathrm{MW}$ as in [18]. As the sensors are moving constantly at $0.08 \mathrm{~m} / \mathrm{s}$, the GPS consumes energy per meter given by following equation:

$$
0.198 \times(1 / 0.08)=2.475 \text { Joules } / \text { meter }
$$

As given in the Figure 3, virtual force algorithm will consume lesser energy as compared to average distance based algorithm. The graph is plotted between average energy consumption and average coverage for both the algorithms. The VFA uses lesser energy as it is GPS enabled, making it easier for the sensor to redeploy themselves faster than the sensors in average distance based algorithm. In VFA sensors take less time to redeploy as they know their positions corresponding to other sensors and also know the locations of rest of the sensors in the field.

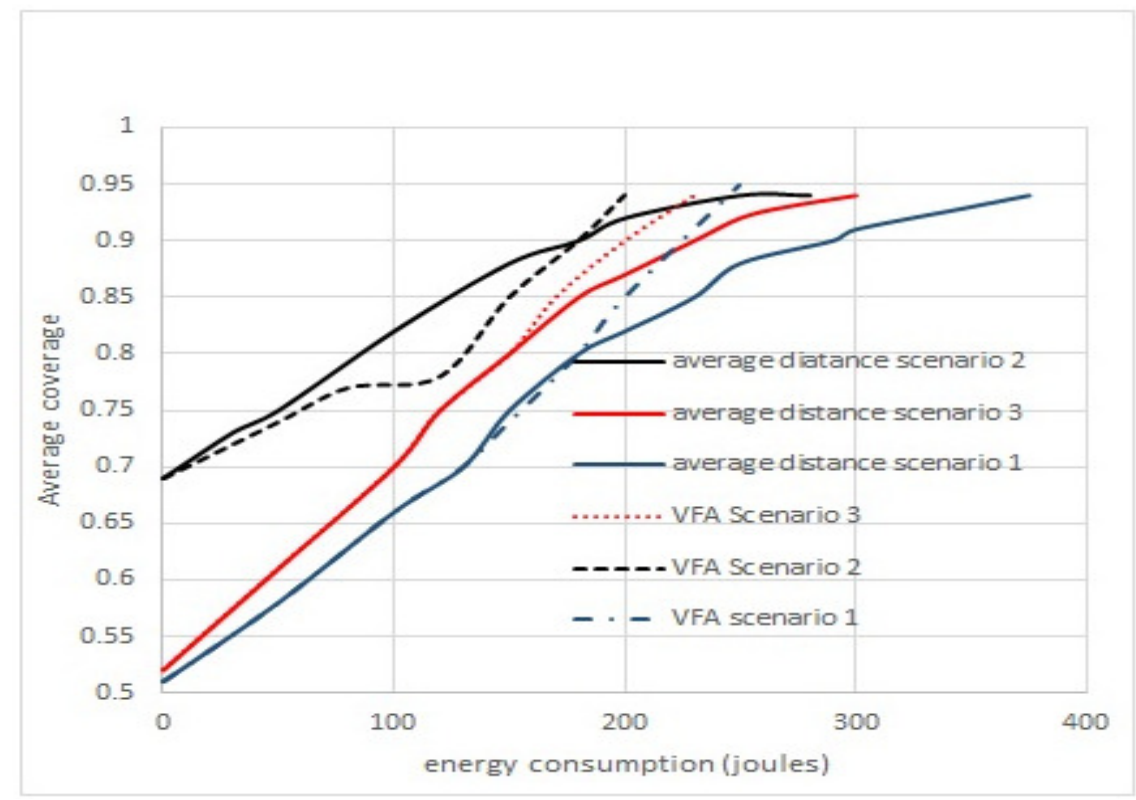

Figure 3: Simulation results for average coverage vs energy consumption

\section{OPTIMIZATION USING AUGMENTED LANGRANGIAN METHOD}

The basic drawback of average distance based algorithm is that it consumes more energy in relocating the sensors as they move back and forth many times to come to an appropriate position to get a good coverage.

Here, if an optimization technique is used to help sensors in their relocation process, they can come to final position in lesser time consuming lesser energy. In the average distance based selfrelocation process, virtual nodes are considered at the boundary and outside the boundary as shown in figure 1 . Using these virtual nodes, the sensors make an idea of their boundary and the area beyond which they are restricted. The sensors move back and forth in self -relocation process, during which the sensors nearer to the boundary have to recalculate its positions to stay 
within the boundary, which can be energy consuming. Thus, in the augmented lagrangian method, the sensors are applied a penalty function. Using this penalty function, if the sensor skips outside the boundary by certain distance $\mathrm{d}_{\text {out }}$, it is made to to come inside the boundary by the same distance $\mathrm{d}_{\mathrm{out}}$, as measured from the boundary. During this process, the sensor might overlap or come into the boundary of another sensor, violating the threshold distance conditions as discussed in section 2.3.

In the augmented lagrangian optimization method, an objective function has to be considered. The minimum distance between the sensors should be $\mathrm{d}_{\mathrm{th}}$, as in average distance based relocation process. This minimum distance might change when penalty function is applied, so minimum distance between sensor i.e. threshold distance $d_{t h}$ is the objective function subject to constraints in augmented lagrangian optimization method.

Now let the boundary of the sensor field be defined by following functions.

$$
g_{1}>0, g_{2} \geq 0, g_{3}<0, \ldots, g_{n}>0
$$

Here, $g_{1}, g_{2}, g_{3}, \ldots, g_{n}$ are the boundary equations for sensor field and $\mathrm{n}$ is the number of boundaries.

In augmented lagrangian optimization method, new objective function is given as

$$
f[X]-\alpha\left\langle d_{t h}\right\rangle-\beta\left\langle g_{1}[X]+g_{2}[X]+\cdots+g_{n}[X]\right\rangle
$$

$[X]$ is the co-ordinate of all the sensors obtained after first iteration of average distance based relocation algorithm. The outline of the algorithm is as follows:

- The $[X]$ co-ordinates of all the sensors in the sensing field are obtained after the execution of first iteration of average distance based relocation algorithm.

- The boundary constraint violations are checked i.e. it is checked if any sensor is lying on the boundary or beyond the boundary. The constraints are checked $n$ times which is equal to the number of sensors in the field.

- If any constraint is violated, let us say for nth sensor, $\left[g_{1}\right]_{i} \nless 0$, which means that constraint $g_{1}$ is violated by ith sensor and the sensor is moving beyond the boundary of sensing field. Hence, the distance $\mathrm{D}_{\mathrm{i}}$ is calculated between $g_{1}$ and $[X, Y]_{i}$. $\mathrm{X}$ and $\mathrm{Y}$ are the co-ordinated of ith sensor lying outside the boundary.

- To compensate for distance and to bring the sensor back inside the field, negative of distance $\mathrm{D}_{\mathrm{i}}$ is added in $\mathrm{g}_{1}$ and the new co-ordinates of ith sensor are calculated.

- Now, all the co-ordinates serve as initial solution for average distance based algorithm which is run again.

- The threshold distance are again checked in the self -relocation algorithm.

Hence, this method is a modified version of average distance based self-relocation algorithm using the properties of augmented lagrangian optimization technique. 


\section{FUTURE WORK}

The biggest advantage of the relocation scheme discussed is non- requirement of a GPS hardware which cuts cost and increases the applicability of this relocation process where GPS cannot be used. The drawback of more energy consumption is to some extent improved by the optimization technique, yet more work can be done to further lessen the energy consumption of non GPS using sensors.

\section{REFERENCES}

[1] V. Potdar, A. Sharif and E. Chang, "Wireless Sensor Networks: A Survey," Advanced Information Networking and Applications Workshops, Bradford, 26-29 May 2009, pp. 636-641.

[2] Ding, W. and Marchionini, G. 1997. A Study on Video Browsing Strategies. Technical Report. University of Maryland at College Park.

[3] J. Chen and C. Li, "Coverage Optimization Based on Improved NSGA-II in Wireless Sensor Network," IEEE International Conference on Integration Technology (ICIT), Shenzhen, 20-24 March 2007, pp. 614-618.

[4] X. Wang, S. Wang and D. W. Bi, "Dynamic Sensor Nodes Selection Strategy for Wireless Sensor Networks," 7th International Symposium on Communications and Information Technologies (ISCIT), Sydney, 16-19 October 2007, pp. 1137-1142.

[5] J. Weck,"Layout Optimization for a Wireless Sensor Network Using a Multi Objective Genetic Algorithm," IEEE 59th Vehicular Technology Conference, Milan, Vol.5, 2004, pp.2466-2470.

[6] L.C. Wei, C. W. Kang and J. H. Chen, "A Force-Driven Evolutionary Approach for Multi-Objective 3D Differentiated Sensor Network Deployment," IEEE 6th International Conference on Mobile Adhoc and Sensor Systems (MASS), Macau, 12-15 October 2009, pp. 983-988

[7] S. Dadwal, T.S. Panag, "Sensor Deployment Strategies in WSNs," 3rd National Conference on Recent Advances in Electronics and Communication Technologies, Ludhiana, 21-22 March 2013, pp 412-419.

[8] A. Howard, M. Mataric and G. Sukhatme, "Mobile Sensor Network Deployment Using Potential Fields: A Distributed, Scalable Solution to the Area Coverage Problem," The 6th International Symposium on Distributed Autonomous Robotics System, Fukuoka, 25-27 June 2002, pp. 299-308.

[9] Yi. Zou and K. Chakrabarty, "Sensor deployment and Target Localization Based on Virtual Forces," IEEE Societies Twenty Second Annual Joint Conference of the IEEE Computer and Communications (INFOCOM), San Fransisco, 1-3 April 2003, pp. 1293-1303

[10] M. R. Pac, A. M. Erkmen and I. Erkmen, "Scalable Self- Deployment of Mobile Sensor Networks: A Fluid Dynamics Approach," Proceedings of IEEE International Conference on Intelligent Robots and Systems (RSJ), Beijing, 9-15 October 2006, pp. 1446-1451.

[11] R.-S. Chang and S.-H. Wang, "Self-Deployment by Density Control in Sensor Networks," IEEE Transactions on Vehicular Technology, Vol. 57, No. 3, 2008, pp. 1745-755.

[12] G. Wang, G. H. Cao, T. F. Porta and W. S. Zhang, "Sensor Relocation in Mobile Sensor Networks," IEEE Societies 24th Annual Joint Conference of the IEEE Computer and Communications, Miami, 2005, pp. 2302-2312.

[13] M. Cardei, J. Wu, M. M. Lu and M. O. Pervaiz, "Maximum Network Lifetime in Wireless Sensor Networks with Adjustable Sensing Ranges, “ IEEE International Conference on Wireless and Mobile Computing, Net- working and Communications, Montreal, 22-24 August 2005, pp. 438-445

[14] Y. Qu, S.V. Georgakopoulos, "An Average Distance Based Self-Relocation and Self-Healing Algorithm for Mobile Sensor Networks," Journal of Wireless Sensor Network, Vol. 4 No.11, pp. 257$263,2012$.

[15] G. Wang, G. H. Cao and T. F. Porta, "Movement-Assisted Sensor Deployment," IEEE Transactions on Mobile Computing, Vol. 5, No. 6, 2006, pp. 640-652. 
[16] W. H. Sheng, G. Tewolde and S. Ci, "Micro Mobile Ro- bots in Active Sensor Networks: Closing the Loop," Proceedings of IEEE International Conference on Intelligent Robots and Systems (RSJ), Beijing, 9-15 October 2006, pp. 1440-1445.

[17] Y. G. Mei, Y.-H. Lu, Y. C. Hu and C. S. G. Lee, "Energy Efficient Motion Planning for Mobile Robots," Proceedings of IEEE International Conference on Robotics and Automation (ICRA), New Orleans, 25 April-1 May 2004, pp. 4344-4349.

[18] M. K. Stojcev, M. R. Kosanovic and L. R. Golubovic, "Power Management and Energy Harvesting Techniques for Wireless Sensor Nodes," 9th International Conference on Telecommunication in Modern Satellite, Cable, and Broadcasting Services, 7-9 October 2009, pp. 65-72.

[19] S. Singh, E. Singla, "Optimal design of robotic arms in constrained environment," TEQIP-II sponsored National conference on 'Latest Developments in Materials, Manufacturing and Quality Control’ MMQC, Bathinda, 18-19 March 2015.

\section{AUTHORS}

Shivani Dadwal had completed her B.Tech degree from RIEIT, Railmajra. Now she is pursuing her M.Tech from BBSEBC, Fatehgarh Sahib.

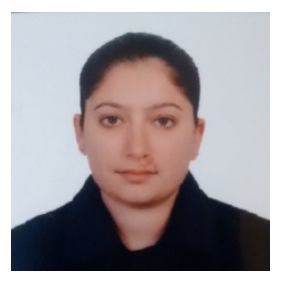

